\title{
Sensitivity and Uncertainty Analysis of the Keff Due to ENDF/B-VII.0 Cross Sections Uncertainties of the Major Isotopes in Nuclear Reactors
}

\author{
M. Kaddour ${ }^{1, *}$, T. El Bardouni ${ }^{1}$, Y. Boulaich ${ }^{2}$, O. Allaoui ${ }^{1}$, B. El Bakkari ${ }^{2}$, C. El Younoussi ${ }^{2}$, M.Azahra ${ }^{1}$, \\ H. Boukhal ${ }^{1}$, S. EL Ouahdani ${ }^{1}$, E. Chakir ${ }^{3}$ \\ ${ }^{1}$ Radiations and Nuclear Systems Laboratory, University Abdelmalek Essaadi, Faculty of Sciences of Tetuan, Morocco \\ ${ }^{2}$ CEN-Maamora, CNESTEN, Rabat, Morocco \\ ${ }^{3}$ Nuclear Physic Laboratory, Faculty of Sciences of Kenitra, Morocco \\ *Corresponding Author: moh-gad@live.fr
}

Copyright (C) 2013 Horizon Research Publishing All rights reserved.

\begin{abstract}
The main objective of this study is to estimate nuclear data uncertainties on the effective multiplication factor (Keff) related to elastic, inelastic, capture and fission cross sections and the correlations between them. Different rapid and thermal cases of the different IHECSBE benchmarks have been studied by using nuclear data evaluation ENDF/B-VII.0 to calculate the sensitivity vectors for ${ }^{1} \mathrm{H},{ }^{16} \mathrm{O},{ }^{235} \mathrm{U}$ and ${ }^{238} \mathrm{U}$ isotopes and four cases used to validate our sensitivity vectors. These sensitivity vectors are calculated by using the adjoint-weighted perturbation method based on the Kpert card of the Monte Carlo code MCNP6. Thus, the uncertainties induced by nuclear data have been calculated by combining the sensitivity vectors with the covariance matrices that are generated by the ERRORJ module of the recently updated of the nuclear data processing system NJOY99. In this study, we found several cross sections and covariance matrices lack the adjustment: the four cross sections (elastic, inelastic, capture and fission) of the ${ }^{235} \mathrm{U}$ and their covariance matrices Lack the adjustement especially in the rapid energies; the elastic cross section of the ${ }^{16} \mathrm{O}$, the elastic and capture cross sections of the ${ }^{1} \mathrm{H}$ and their covariance matrices lack the adjustement especially in the thermal energies.
\end{abstract}

Keywords Cross Section, Sensitivity, Covariance Matrix, Nuclear Uncertainty, MCNP6

\section{Introduction}

Since the beginning of the century, the nuclear data evaluation communities are putting more and more attention to the assessment of uncertainties. This increased interest concerns both basic data (cross section, emission spectrum ...) and calculated quantities for large systems. Such as neutron multiplication factor Keff, reactivity, reaction rate and others.

With the large availability of the covariance files, as in the ENDF/B-VII.0 [1] nuclear data evaluation, more and more studies are started using this information to deduce target accuracies for neutronic parameters in future reactors and therefore future priorities for experimental measurements of differential data.

Several probabilistic and deterministic codes are made for the analysis of the sensitivity and the uncertainties of the nuclear data on the integral nuclear parameters (SCALE, MCNP, KENO, DRAGON...). Most of these codes used the multigroup adjoint-weighted technique. Except MCNP6 [2] computed the changes in a tally response (such as Keff-eigenvalue) by using the differential operator technique with continuous energy spectrum [3]. Now, it compute the changes in reactivity strictly in Keff-eigenvalue problems by using the adjoint-weighted methodology with continuous energy spectrum [4,5]. The technique of differential operator considered the source of neutrons unperturbed [6]. The sensitivity with continuous energy spectrum has no problem of the self shielding effect than the sensitivity with multigroup energy spectrum [7,8]. For this, we used the adjoint-weighted technique with continuous energy spectrum to calculate the sensitivity profiles in this work. The sensitivity profiles and covariance matrices are combined in order to obtain final uncertainties [9].

Several sensitivities and uncertainties analysis for improving and adjusting the cross sections of the important isotopes in nuclear data evaluation ENDF/B-VII.0 and in simple and complex nuclear systems are done by different codes and different perturbation techniques [10, 11]. But, there are still errors on the integral nuclear parameters due to cross sections uncertainties of the ${ }^{235} \mathrm{U},{ }^{16} \mathrm{O}$ and ${ }^{1} \mathrm{H}$ in ENDF/B.VII.0. These errors are detected with the new adjoint-weighted technique of the MCNP6 code.

In this work, a sensitivity and uncertainty analysis have 
been performed for certain cases of the different IHECSBE (International Handbook of Evaluated Criticality Safty Benchmark Experiments) [12] benchmarks by using the MCNP6 and NJOY99 [13] codes. Results of the uncertainty calculated by the adjoint-weighted theory and produced by elastic, inelastic, capture and fission nuclear data on the multiplication factor are presented and analyzed.

\section{Methodology and Study Approach}

\subsection{Adjoint-weighted Technique}

Starting from the nuclear transport equation and applying a first-order perturbation, the following expression for the change in reactivity $\rho$ can be derived [4]:

$$
\left.\Delta \boldsymbol{\rho}=-\frac{\left\langle\boldsymbol{\Psi}^{+}, \mathbf{P} \boldsymbol{\psi}\right\rangle}{\left\langle\boldsymbol{\Psi}^{+}, \mathbf{F}^{\prime}\right.} \boldsymbol{\psi}\right\rangle
$$

The reactivity is related to Keff in the typical way:

$$
\boldsymbol{\rho}=\frac{(\text { Keff }-\mathbf{1})}{\text { Keff }}
$$

The angular flux in the unperturbed system is $\psi$ and its adjoint is denoted by $\boldsymbol{\psi}^{+}$. $\mathrm{P}$ is the operator for the perturbation taking the form:

$$
\boldsymbol{P}=\Delta \Sigma_{t}-\Delta \boldsymbol{S}-\lambda \Delta \boldsymbol{F}
$$

The eigenvalue $\lambda$ is:

$$
\lambda=\frac{1}{\text { Keff }}
$$

And the tree terms in $\boldsymbol{P}$ from left to right, are the change in the total cross section, the change in scattering operator, and the change in the fission multiplication operator. F' is the perturbed fission operator.

Monte Carlo technique can be used to sample the numerator and the denominator in continuous-energy forward calculation [4] and the change in reactivity can be estimated by taking the ratio in (1).

We express change in cross section as:

$$
\Delta \sigma_{x}=f \sigma_{x}
$$

We apply the relationship:

$$
\Delta K_{e f f}=K_{e f f} \frac{K_{e f f} \Delta \rho}{1-K_{e f f} \Delta \rho}
$$

We compute sensitivity coefficients by:

$$
S_{K_{e f f, \sigma_{x}}} \approx \frac{1}{f} \frac{K_{\text {eff }} \Delta \rho}{1-K_{\text {eff }} \Delta \rho}
$$

The quantity $\boldsymbol{K}_{\boldsymbol{e f f}} \Delta \boldsymbol{\rho}$ scales linearly with f; can make arbitrarily small until sensitivity becomes sufficiently precise.

\subsection{Study Approach}

During this study:

We have selected a different criticality safety cases of IHECSBE [12] benchmarks.
We have studied the impact of the cross sections uncertainties for ${ }^{1} \mathrm{H},{ }^{16} \mathrm{O},{ }^{235} \mathrm{U}$, and ${ }^{238} \mathrm{U}$ isotopes on the effective multiplication factor uncertainty in each selected case. The selected isotopes are generally known by their important contribution in the effective multiplication factor

\begin{tabular}{|c|c|}
\hline Groups numbers & Energy groups (Mev) \\
\hline 1 & $0.00 \mathrm{E} 00-1.10 \mathrm{E}-07$ \\
\hline 2 & $1.10 \mathrm{E}-07-5.40 \mathrm{E}-07$ \\
\hline 3 & $5.40 \mathrm{E}-07-4.00 \mathrm{E}-06$ \\
\hline 4 & $4.00 \mathrm{E}-06-2.26 \mathrm{E}-05$ \\
\hline 5 & $2.26 \mathrm{E}-05-4.54 \mathrm{E}-04$ \\
\hline 6 & 4.54E-04 - 2.04E-03 \\
\hline 7 & $2.04 \mathrm{E}-03-9.12 \mathrm{E}-03$ \\
\hline 8 & $9.12 \mathrm{E}-03-2.48 \mathrm{E}-02$ \\
\hline 9 & $2.48 \mathrm{E}-02-6.74 \mathrm{E}-02$ \\
\hline 10 & $6.74 \mathrm{E}-02-1.83 \mathrm{E}-01$ \\
\hline 11 & $1.83 \mathrm{E}-01-4.98 \mathrm{E}-01$ \\
\hline 12 & $4.98 \mathrm{E}-01-1.35 \mathrm{E} 00$ \\
\hline 13 & $1.35 \mathrm{E} 00-2.23 \mathrm{E} 00$ \\
\hline 14 & $2.23 \mathrm{E} 00-6.07 \mathrm{E} 00$ \\
\hline 15 & $6.07 \mathrm{E} 00-19.60 \mathrm{E} 00$ \\
\hline
\end{tabular}
calculation.

The sensitivity vectors for multiplication factor were generated by using Kpert card of MCNP6 code in 15 energy groups, they are represented in table 1 .

Table 1. Fifteen energy groups used in our sensitivity and uncertainty analysis

The covariance matrices generated by ERRORJ module of the NJOY99 [13] processing system based on the same discretization of the sensitivity energy. The values of the covariance were computed for fifteen energy groups mentioned above by using a weighting flux that corresponds to the $\mathbf{1} / \boldsymbol{E}+$ fission spectrum + thermal maxwellian shape. For all cases, an infinite dilution condition was assumed $\left(\sigma_{0}=\mathbf{1} \cdot \mathbf{1 0}^{\mathbf{1 0}}\right.$ barns $)$ and the temperature was considered to be $300 \mathrm{~K}$.

The steps adopted in this study are presented in Figure 1:

- The perturbation approach is generally based on the NJOY99 processing system, the MCNP6 code and the program for calculating the total uncertainty produced by nuclear data ( $\triangle$ Keff-nucl.).

- Inputs are the MCNP6 input file and ENDF/B-VII.0 file containing the matrices of covariances. As shown in figure 1, ENDF/B-VII.0 file is processed by NJOY99 in order to produce cross sections in the ACE format and covariance matrices used by program for calculating $\Delta$ Keff-nucl.

- The sensitivity vectors were calculated by using the most commonly used radiation transport code MCNP6. The sensitivity profiles $\boldsymbol{S}_{\boldsymbol{K} \boldsymbol{e f f}, \boldsymbol{\sigma}_{\boldsymbol{x}, \boldsymbol{g}}}$ are defined as the relative 
change in a response Keff (the effective multiplication factor) with respect to cross section $\sigma_{x}$ in a particular energy group $\mathrm{g}$, it is defined as:

$$
S_{K e f f, \sigma_{x}, g}=\frac{\sigma_{x, g}}{K e f f} \frac{\Delta K e f f}{\Delta \sigma_{x, g}} \approx \frac{1}{f} \frac{K_{e f f} \Delta \rho}{1-K_{e f f} \Delta \rho}
$$

- The sensitivity profile $\boldsymbol{S}_{\boldsymbol{K} e f f, \sigma_{x, g}}$ is obtained by using the perturbation option of MCNP6 that is defined in Kpert card by using the first-order perturbation by the adjoint-weighted technique.

- The cross sections were perturbed as described in the following steps:

1. Four cross sections will be considered: elastic, inelastic, capture and fission cross section, and only one specific cross section in one energy group and one isotope varied each time.

2. Then a material card is created in which the atomic density for the relevant isotope is increased by $\mathbf{1} \%$.

3. The Kpert card is then created specifying that: the relevant material is replaced by the perturbed material in each of the cells in which the material is present. Perturbation cards are given for all energy groups.

4. Finally, MCNP6 is run with this modification in the input; and in the output file a table is given with the results of different perturbations and their related statistical uncertainties.

- These sensitivity vectors must be combined with covariances matrices (10) and (11), by using a program calculating Keff uncertainties in similar energy groups.

- The total uncertainty is calculated by using the following equation [9]:

$$
\frac{\Delta \text { Keff }}{\text { Keff }}=\sqrt{\sum_{i} \sum_{\sigma_{x}} \sum_{g}\left[\frac{\Delta \text { Keff }}{\text { Keff }}\right]_{i, \sigma_{x}, g}^{2}}
$$

i: index of material

$\sigma_{x}$ : index of reaction cross section

$\mathrm{g}$ : index of energy group

- The contribution of every nuclide-reaction in $\frac{\Delta \text { Keff }}{\text { Keff }}$ is calculated as follows [5]:

$$
\left\{\begin{array}{l}
\sqrt{S_{K e f f, \sigma_{x, g}} \operatorname{cov}\left(\sigma_{x, g}, \sigma_{y, g^{\prime}}\right) S_{K e f f, \sigma_{y, g^{\prime}}}} \\
-\sqrt{\mid S_{K e f f, \sigma_{x, g}} \operatorname{cov}\left(\sigma_{x, g}, \sigma_{y, g^{\prime}}\right) S_{K e f f, \sigma_{y, g^{\prime}} \mid}}
\end{array}\right.
$$

If $\left.\left(\boldsymbol{S}_{K e f f, \sigma_{x, g}} \operatorname{cov}\left(\sigma_{x, g^{\prime}}, \sigma_{y, g^{\prime}}\right) \boldsymbol{S}_{K e f f, \sigma_{y, g^{\prime}}}\right) \geq 0\right)$, we have using the equation (10).

If $\left(\boldsymbol{S}_{K e f f, \sigma_{x, g}} \operatorname{cov}\left(\sigma_{x, g}, \sigma_{y, g^{\prime}}\right) \boldsymbol{S}_{K e f f, \sigma_{y, g^{\prime}}}<0\right)$, we have using the equation (11).

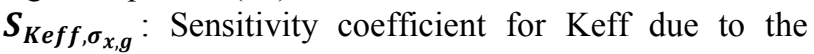
neutron cross section $\boldsymbol{\sigma}_{\boldsymbol{x}}$, and energy group $\mathrm{g}$.

$\operatorname{cov}\left(\sigma_{x, g}, \sigma_{y, g^{\prime}}\right)$ : Covariance matrix that comprises covariance data for two cross sections $\left(\sigma_{x}, \sigma_{y}\right)$ in the energy groups $\mathrm{g}$ and $\mathrm{g}$ '.

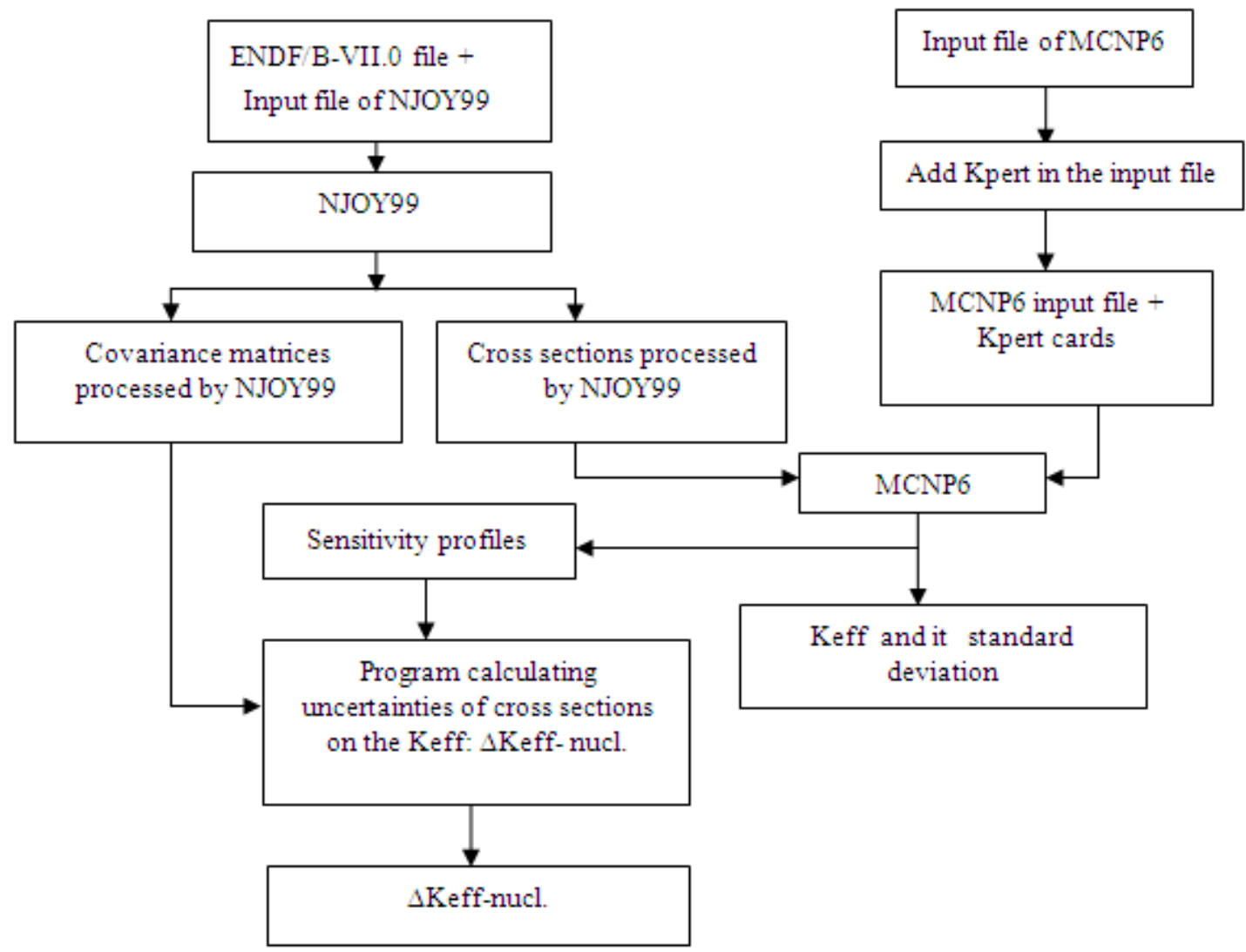

Figure 1. Flowchart of the uncertainty calculation by MCNP6 and NJOY99 codes 


\section{Effective Multiplication Factors Calculated by MCNP6}

The $\mathrm{K}_{\text {eff }}$ values with their related standard deviations for the cases studied are listed in table 2. The third column represent values from the International Criticality Safty Benchmark experements (IHECSBE). Our results calculated by MCNP6 code and ENDF/B-VII.0 nuclear data evaluation are represented in the second column.

We chose these cases because their relative differences between the experimental Keff and the calculated Keff are higher than their experimental uncertainties and their standard deviations.

Table 2. HEU-SOL-THERM-001-cases Keff and its standard deviation

\begin{tabular}{|c|c|c|}
\hline Cases of benchmarks & $\begin{array}{c}\text { Keff } \pm \text { std. dev. } \\
\text { (MCNP6 })\end{array}$ & $\begin{array}{c}\text { Keff } \pm \Delta \text { Keff }^{2} \\
\text { (IHECSBE) }\end{array}$ \\
\hline $\begin{array}{c}\text { HEU-MET-FAST-004-001 } \\
\text { (hmf004-001) }\end{array}$ & $0.99987 \pm 0.00023$ & $0.99850 \pm \mathbf{0 . 0 0 0 0}$ \\
\hline $\begin{array}{c}\text { HEU-MET-FAST-008-001 } \\
\text { (hmf008-001) }\end{array}$ & $0.99615 \pm 0.00019$ & $0.99890 \pm \mathbf{0 . 0 0 1 6}$ \\
\hline $\begin{array}{c}\text { HEU-MET-FAST-015-001 } \\
\text { (hmf015-001) }\end{array}$ & $0.99445 \pm 0.00019$ & $0.99960 \pm \mathbf{0 . 0 0 1 7}$ \\
\hline $\begin{array}{c}\text { HEU-SOL-THERM-006-001 } \\
\text { (hst006-001) }\end{array}$ & $0.98229 \pm 0.00031$ & $0.99730 \pm 0.0050$ \\
\hline $\begin{array}{c}\text { HEU-SOL-THERM-011-001 } \\
\text { (hst011-001) }\end{array}$ & $1.00466 \pm 0.00025$ & $1.00000 \pm 0.0023$ \\
\hline $\begin{array}{c}\text { HEU-SOL-THERM-016-001 } \\
\text { (hst016-001) }\end{array}$ & $0.99022 \pm 0.00036$ & $1.00000 \pm 0.0036$ \\
\hline $\begin{array}{c}\text { HEU-SOL-THERM-018-001 } \\
\text { (hst018-001) }\end{array}$ & $0.98739 \pm 0.00040$ & $1.00000 \pm 0.0034$ \\
\hline $\begin{array}{c}\text { HEU-SOL-THERM-028-001 } \\
\text { (hst028-001) }\end{array}$ & $0.99640 \pm 0.00031$ & $1.00000 \pm 0.0023$ \\
\hline $\begin{array}{c}\text { HEU-SOL-THERM-035-007 } \\
\text { (hst035-007) }\end{array}$ & $1.00540 \pm 0.00034$ & $1.00000 \pm 0.0035$ \\
\hline HEU-SOL-THERM-037-001 \\
(hst037-001)
\end{tabular}

${ }^{1}$ This uncertainty means the statistical uncertainty or standard deviation in MCNP6 calculated with the Monte Carlo technique.

${ }^{2}$ This uncertainty means the experimental uncertainty due to uncertainties in critical heights, uncertainties in solution constituents, and in isotropic constituents [12]

\section{Validation of Sensitivity Results}

The calculation of the sensitivities coefficients by the adjoint-weighted technique is new in the code MCNP6; for this, we compared the results of this technique with the results of the old technique in MCNP code (differential operator technique) and with the sensitivities coefficients which are in IHECSBE and calculated by the KENO code. Also, we compared the results of the adjoint-weighted technique between tree nuclear data evaluations ENDFB/VI.8, ENDFB/VII.0 and JENDL-4.0 [14]. This comparison is used in 30 energy groups, they are represented in table 3.

Table 3. Thirty energy groups used in our sensitivity validation

\begin{tabular}{|c|c|}
\hline Groups numbers & Energy groups (Mev) \\
\hline 1 & $0.00 \mathrm{E}+0-1.00 \mathrm{E}-02$ \\
\hline 2 & $1.00 \mathrm{E}-02-2.15 \mathrm{E}-02$ \\
\hline 3 & $2.15 \mathrm{E}-02-4.64 \mathrm{E}-02$ \\
\hline 4 & 4.64E-02 - 1.00E-01 \\
\hline 5 & $1.00 \mathrm{E}-01-2.15 \mathrm{E}-01$ \\
\hline 6 & $2.15 \mathrm{E}-01-4.64 \mathrm{E}-01$ \\
\hline 7 & $4.64 \mathrm{E}-01-1.00 \mathrm{E}+00$ \\
\hline 8 & $1.00 \mathrm{E}+00-2.15 \mathrm{E}+00$ \\
\hline 9 & $2.15 \mathrm{E}+00-4.64 \mathrm{E}+00$ \\
\hline 10 & $4.64 \mathrm{E}+00-1.00 \mathrm{E}+01$ \\
\hline 11 & $1.00 \mathrm{E}+01-2.15 \mathrm{E}+01$ \\
\hline 12 & $2.15 \mathrm{E}+01-4.64 \mathrm{E}+01$ \\
\hline 13 & $4.64 \mathrm{E}+01-1.00 \mathrm{E}+02$ \\
\hline 14 & $1.00 \mathrm{E}+02-2.15 \mathrm{E}+02$ \\
\hline 15 & $2.15 \mathrm{E}+02-4.64 \mathrm{E}+02$ \\
\hline 16 & $4.64 \mathrm{E}+02-1.00 \mathrm{E}+03$ \\
\hline 17 & $1.00 \mathrm{E}+03-2.15 \mathrm{E}+03$ \\
\hline 18 & $2.15 \mathrm{E}+03-4.64 \mathrm{E}+03$ \\
\hline 19 & $4.64 \mathrm{E}+03-1.00 \mathrm{E}+04$ \\
\hline 20 & $1.00 \mathrm{E}+04-2.15 \mathrm{E}+04$ \\
\hline 21 & $2.15 \mathrm{E}+04-4.64 \mathrm{E}+04$ \\
\hline 22 & $4.64 \mathrm{E}+04-1.00 \mathrm{E}+05$ \\
\hline 23 & $1.00 \mathrm{E}+05-2.00 \mathrm{E}+05$ \\
\hline 24 & $2.00 \mathrm{E}+05-4.00 \mathrm{E}+05$ \\
\hline 25 & $4.00 \mathrm{E}+05-8.00 \mathrm{E}+05$ \\
\hline 26 & $8.00 \mathrm{E}+05-1.40 \mathrm{E}+06$ \\
\hline 27 & $1.40 \mathrm{E}+06-2.50 \mathrm{E}+06$ \\
\hline 28 & $2.50 \mathrm{E}+06-4.00 \mathrm{E}+06$ \\
\hline 29 & $4.00 \mathrm{E}+06-6.50 \mathrm{E}+06$ \\
\hline 30 & $6.50 \mathrm{E}+06-6.50 \mathrm{E}+06$ \\
\hline
\end{tabular}

The figures below show the results of the sensitivity validation in rapid experiment: Godiva (highly enriched uranium sphere), hmf004-001 and hmf018-001, and in thermal experiment hst001-001; for four reactions: elastic and inelastic scattering, capture and fission.

The differences between the results of sensitivities in the above figures $(2,3,4,5)$ are due to:

- The difference between the nuclear data evaluation used. - The difference between the perturbation techniques used: in the differential operator technique, the fundamental eigenfuction (fission distribution) approximated as unperturbed.

- The absence of thermal neutron scattering: $\mathbf{S}(\boldsymbol{\alpha}, \boldsymbol{\beta})$ in the KENO code.

- The difference between the two codes: MCNP6 uses continuous energy, KENO uses multigroup energy.

In general, the adjoint-weighted technique has the same allure with other technique. Then, it is validated. 


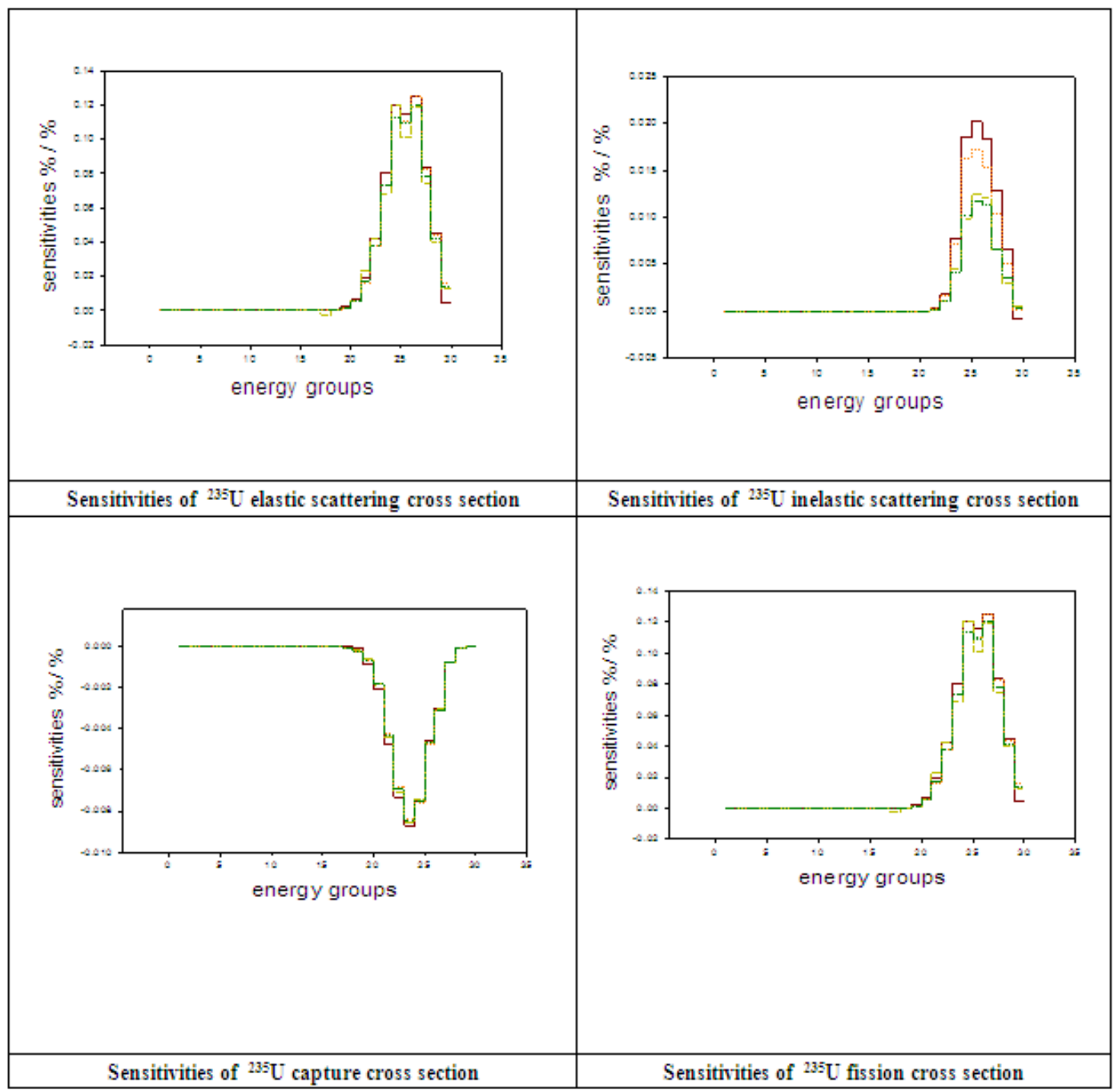

\begin{tabular}{|ll|}
\hline & handbook (KENO) \\
differentiel operator technique ENDF/B-VI.8 \\
$-\cdots \cdots \cdots \cdots \cdots$ \\
$-\cdots-\cdots-\cdots$ & adjoint-weighted technique ENDF/B-VII.0 \\
$-\cdot-\cdot$ adjoint-weighted technique ENDF/B-VI.8
\end{tabular}

Figure 2. Sensitivities of $235 \mathrm{U}$ elastic and inelastic scattering, capture and fission cross sections in Godiva 


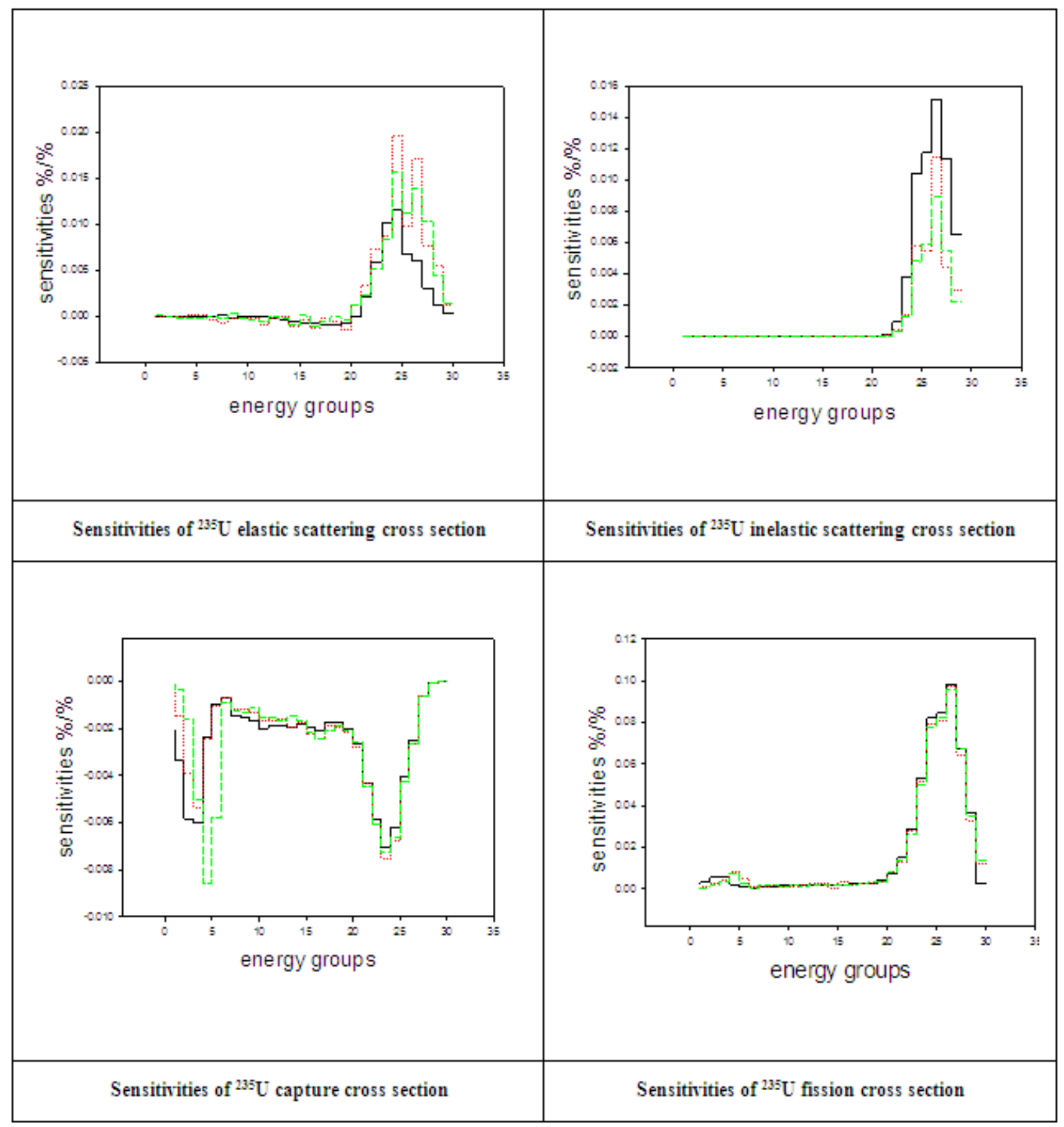

handbook (KENO)

adjoint-weighted technique ENDF/B-VII.0 adjoint-weighted technique ENDF/B-VI.8

Figure 3. Sensitivities of ${ }^{235} \mathrm{U}$ elastic and inelastic scattering, capture and fission cross sections in hmf004-001 


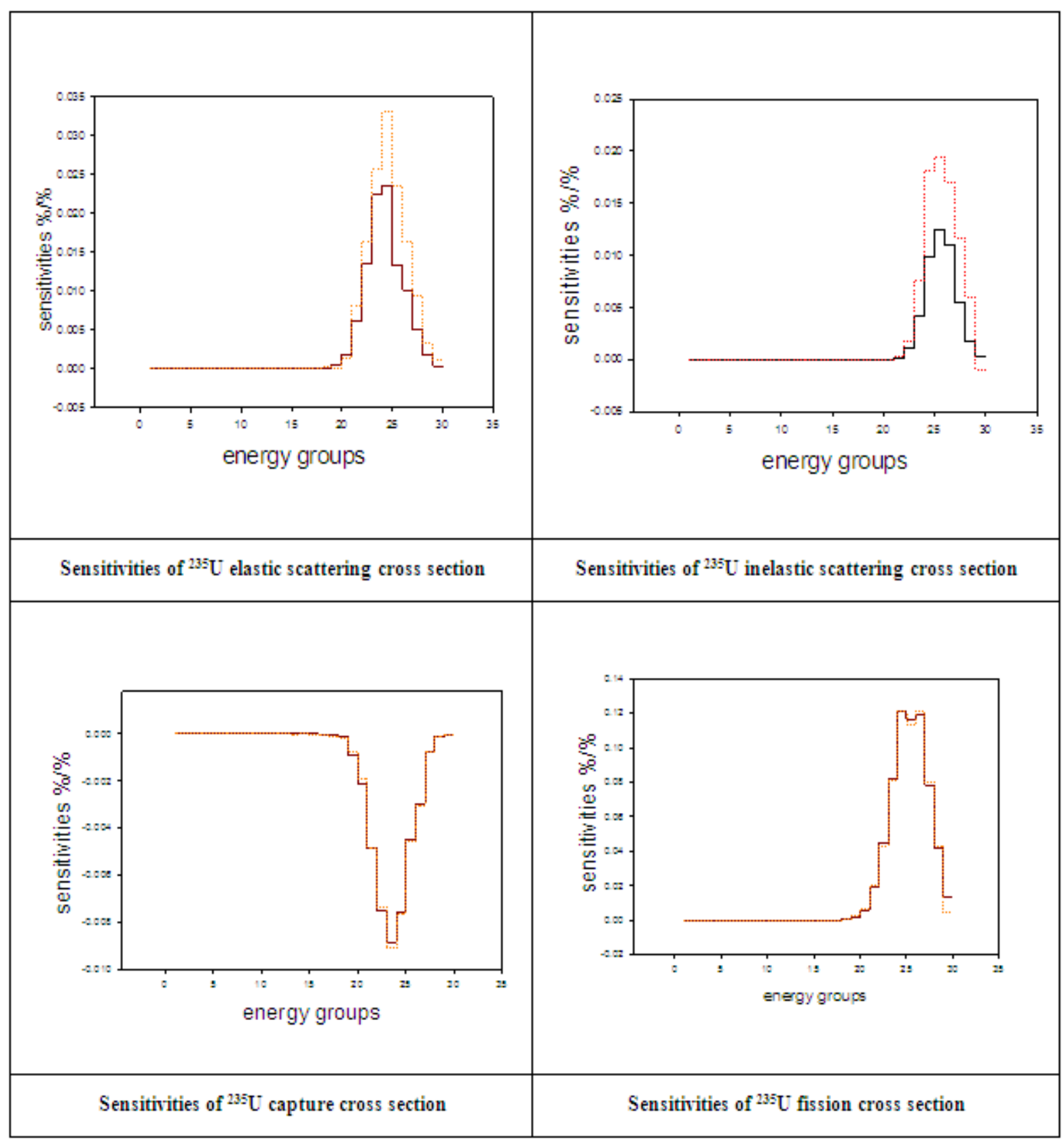

handbook (KENO) adjoint-weighted technique ENDF/B-VII.0

Figure 4. Sensitivities of ${ }^{235} \mathrm{U}$ elastic and inelastic scattering, capture and fission cross sections in hmf018-001 


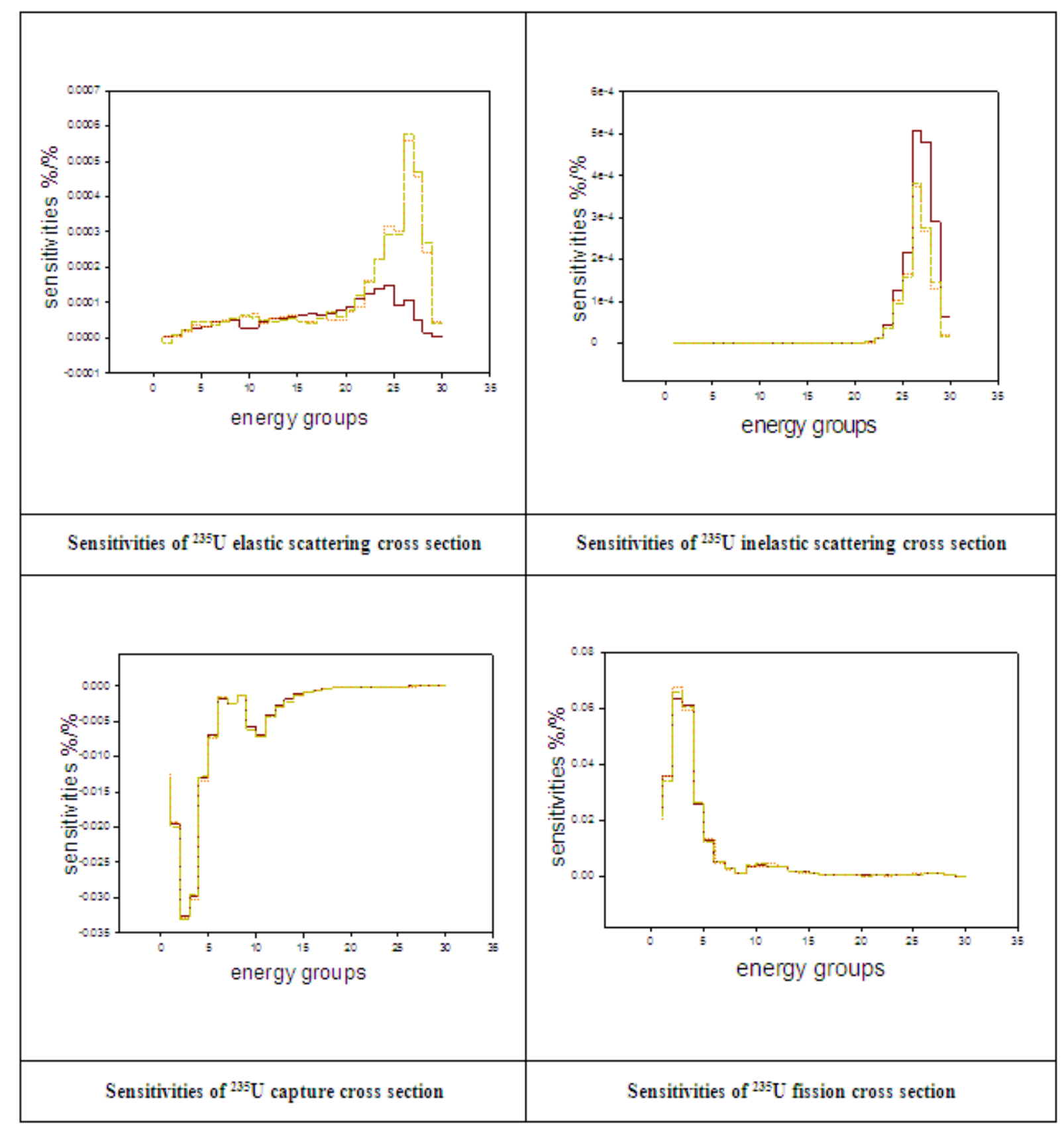

handbook (KENO)

adjoint-weighted technique JENDL-4.0 adjoint-weighted technique ENDF/B-VII.0

Figure 5. Sensitivities of ${ }^{235} \mathrm{U}$ elastic and inelastic scattering, capture and fission cross sections in hst001-001 

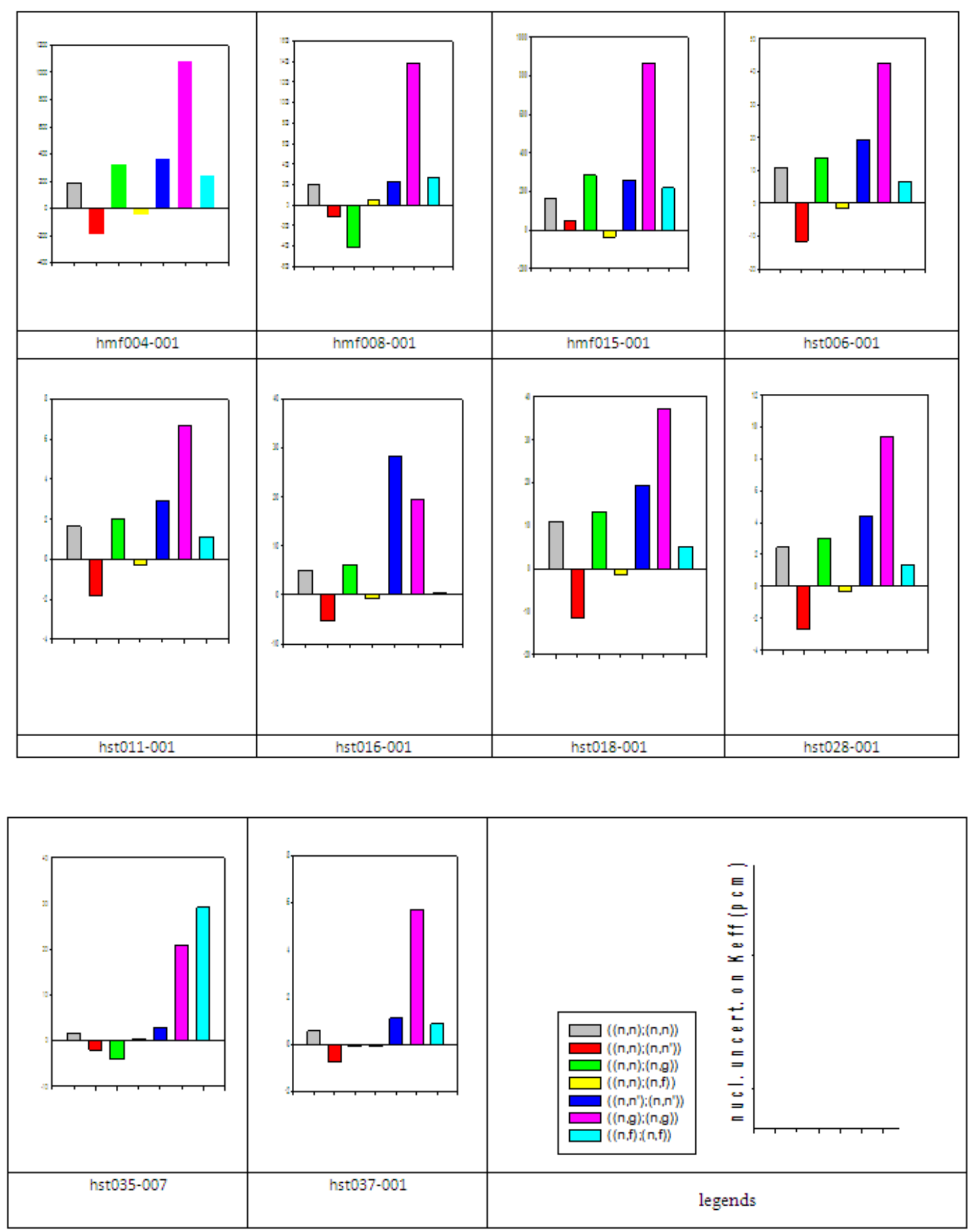

Figure 6. Uncertainties (pcm) produced by different cross sections of ${ }^{235} \mathrm{U}$ for different experiences 


\section{Uncertainty Analysis}

\subsection{Uncertainty on the Keff Produced by the Nuclear Data Uncertainties of the ${ }^{235} \mathbf{U}$}

Figure 6 shows the effect of cross sections uncertainties related to ${ }^{235} \mathrm{U}$ isotope on the effictive multiplication factor (nucl. uncert. on Keff or $\Delta$ Keff-nucl.) in different nuclear experiences. The uncertainties on the Keff produced by elastic, inelastic scattering, capture and fission cross sections and the effect of the correlation between them are represented by adjoint-weighted technique and by $\mathrm{pcm}$ $\left(1 \mathrm{pcm}=10^{-5}\right)$

\subsection{Interpretations and Conclusions on the Results of ${ }^{235} \mathbf{U}$}

- The elastic, inelastic, capture and fission cross sections and the correlations between them for ${ }^{235} \mathrm{U}$ in the rapid experiences, they have large contribution of uncertainties in the effective multiplication factor uncertainties ( $\Delta$ Keff-nucl.). Then, these four cross sections and their covariance matrices in ${ }^{235} \mathrm{U}$ require the adjustment in the rapid energies.

- The elastic, inelastic, capture and fission cross sections uncertainties and the correlations between them for ${ }^{235} \mathrm{U}$ in the thermal experiences, they have small contribution of uncertainties in the effective multiplication factor uncertainties ( $\Delta$ Keff-nucl.) than its contribution of uncertainties in $\Delta \mathrm{Keff}$-nucl of the rapid experiences. But, in another study we found $\Delta \mathrm{Keff}$-nucl. increases with small increase in the atomic density for ${ }^{235} \mathrm{U}$ in six cases of hst 001 [15]. Thus, we cannot assure the accuracy of these cross sections and their covariances matrices in the thermal energies when the ${ }^{235} \mathrm{U}$ atomic density increases.

\subsection{Uncertainty on the Keff Produced by the Nuclear Data Uncertainties of the ${ }^{238} U$}

Figure 7 shows the effect of cross sections uncertainties related to ${ }^{238} \mathrm{U}$ isotope on the effictive multiplication factor (nucl. uncert. on Keff or $\Delta$ Keff-nucl.) in different nuclear experiences. The uncertainties on the Keff produced by elastic, inelastic scattering, capture and fission cross sections and the effect of the correlation between them are represented by adjoint-weighted technique and by $\mathrm{pcm}$ $\left(1 \mathrm{pcm}=10^{-5}\right)$.

\subsection{Interpretations and Conclusions on the Results of ${ }^{238} \mathbf{U}$}

- The effect of elastic, inelastic, capture and fission cross sections uncertainties related to ${ }^{238} \mathrm{U}$ on the effictive multiplication factor uncertainties is very small in the thermal experiences than the rapid experiences; and that is due to the small contribution of ${ }^{238} U$ cross sections on the Keff in the thermal energies. Then, in this study, we can conclude that: these cross sections and their covariance matrices do not lack the adjustment in the thermal energies.

- In another study we found $\Delta$ Keff-nucl. increases with the increase in the ${ }^{238} \mathrm{U}$ atomic density in six cases of hst001 [15]. Thus, we cannot assure the accuracy of these cross sections and their covariance matrices in the rapid energies when the atomic density of the ${ }^{238} \mathrm{U}$ increases.

\subsection{Uncertainty on the Keff Produced by the Nuclear Data Uncertainties of the ${ }^{16} \mathrm{O}$}

Figure 8 shows the effect of cross sections uncertainties related to ${ }^{16} \mathrm{O}$ isotope on the effictive multiplication factor (nucl. uncert. on Keff or $\Delta$ Keff-nucl.) in different nuclear experiences. The uncertainties on the Keff produced by elastic, inelastic scattering, capture and fission cross sections and the effect of the correlation between them are represented by adjoint-weighted technique and by $\mathrm{pcm}$ $\left(1 \mathrm{pcm}=10^{-5}\right)$.

\subsection{Interpretation and Conclusion on the Results of ${ }^{16} \mathrm{O}$}

The cross sections uncertainties have no effect on the effective multiplication factor (Keff) in all experiences, except the elastic scattering cross section uncertainty has the great contribution on the Keff uncertainties in the thermal experiences and in one rapid experience. Then, the elastic scattering cross section and its covariance matrix lack the adjustment especially in the thermal experiences.

\subsection{Uncertainty on the Keff Produced by the Nuclear Data Uncertainties of the ${ }^{1} \mathbf{H}$}

Figure 9 shows the effect of cross sections uncertainties related to ${ }^{1} \mathrm{H}$ isotope on the effictive multiplication factor (nucl. uncert. on Keff or $\Delta$ Keff-nucl.) in different nuclear experiences. The uncertainties on the Keff produced by elastic, inelastic scattering, capture and fission cross sections and the effect of the correlation between them are represented by adjoint-weighted technique and by $\mathrm{pcm}$ $\left(1 \mathrm{pcm}=10^{-5}\right)$. 

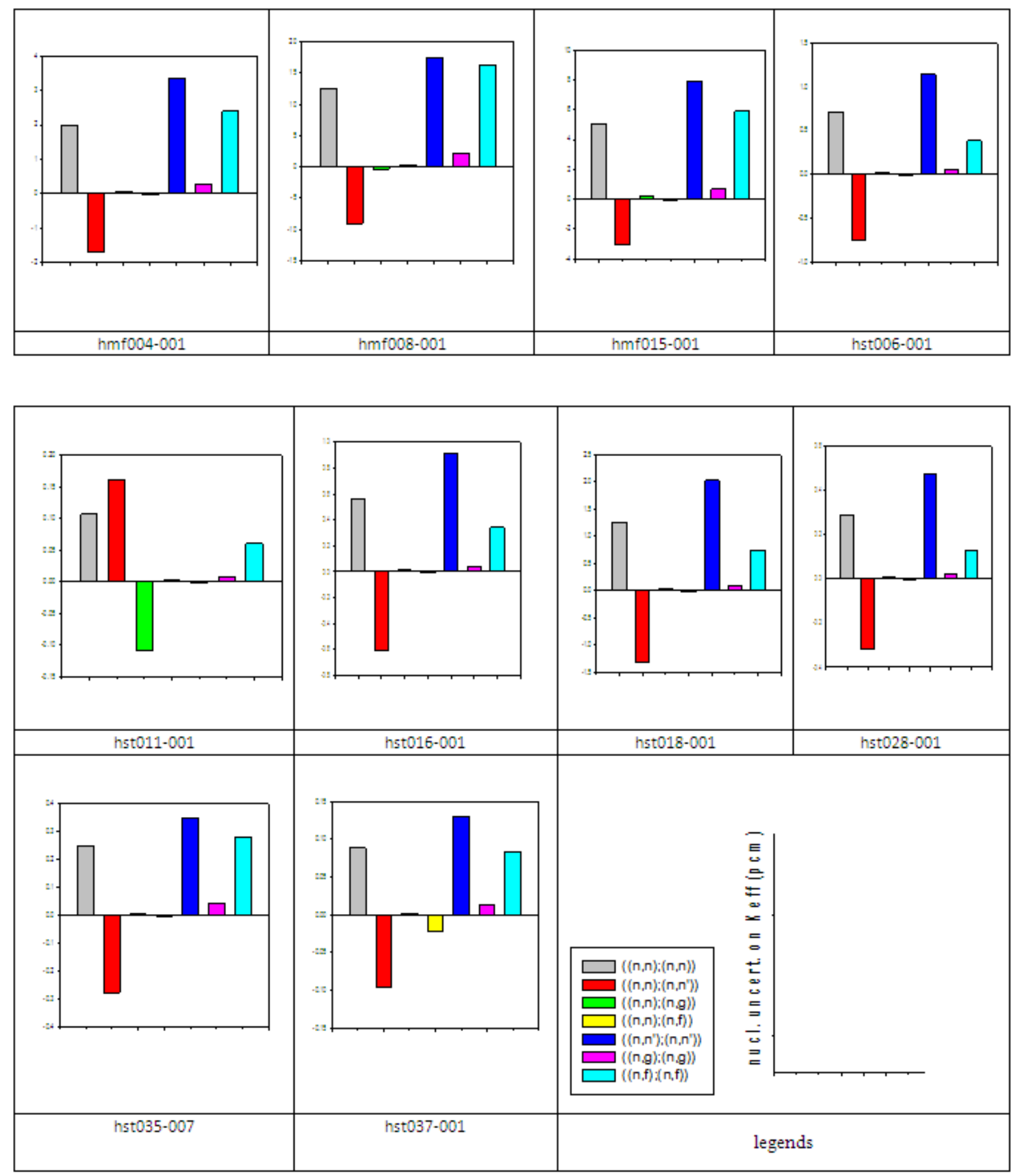

Figure 7. Uncertainties (pcm) produced by different cross sections of ${ }^{238} \mathrm{U}$ for different experiences 


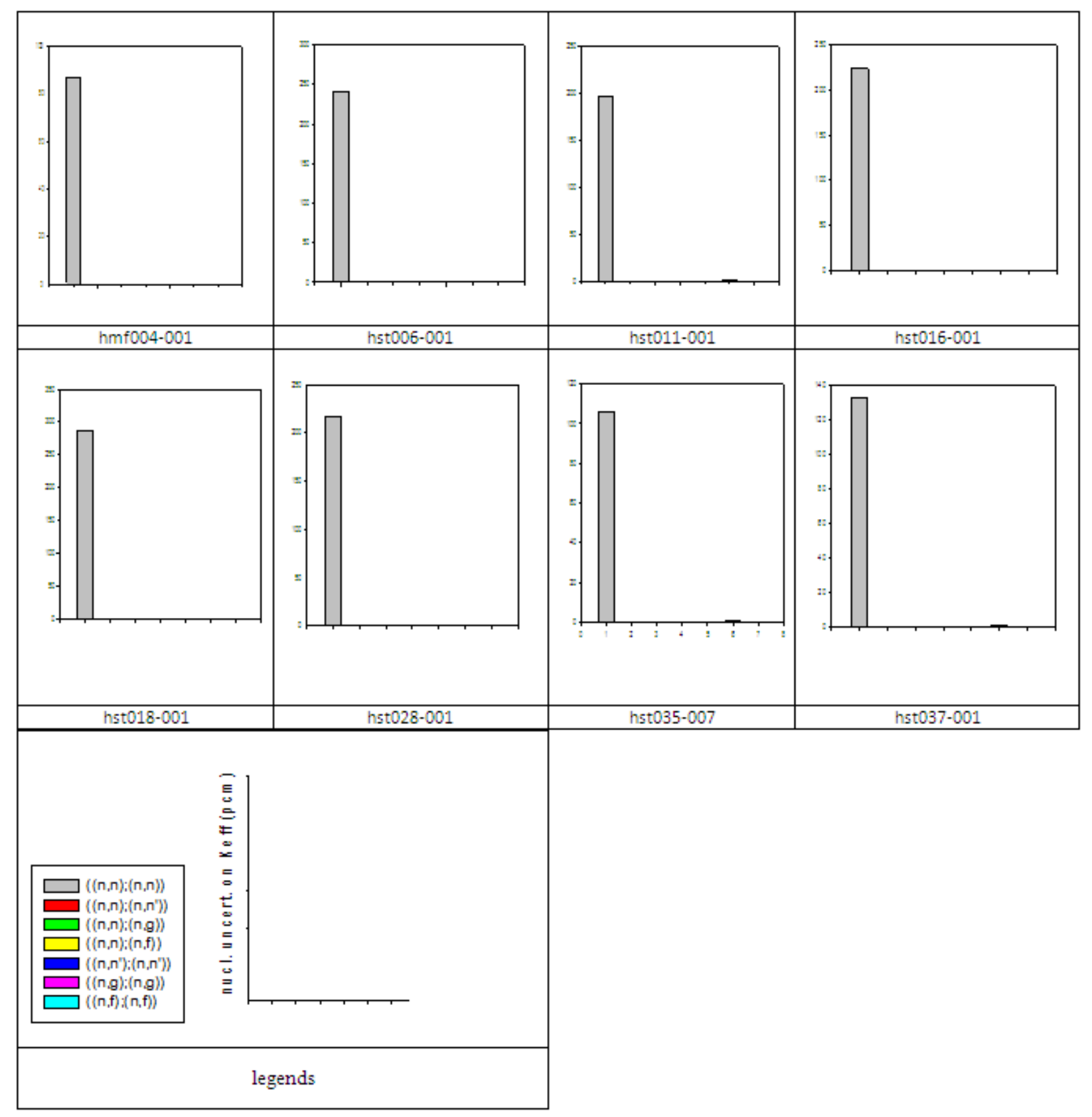

Figure 8. Uncertainties (pcm) produced by different cross sections of ${ }^{16} \mathrm{O}$ for different experiences 


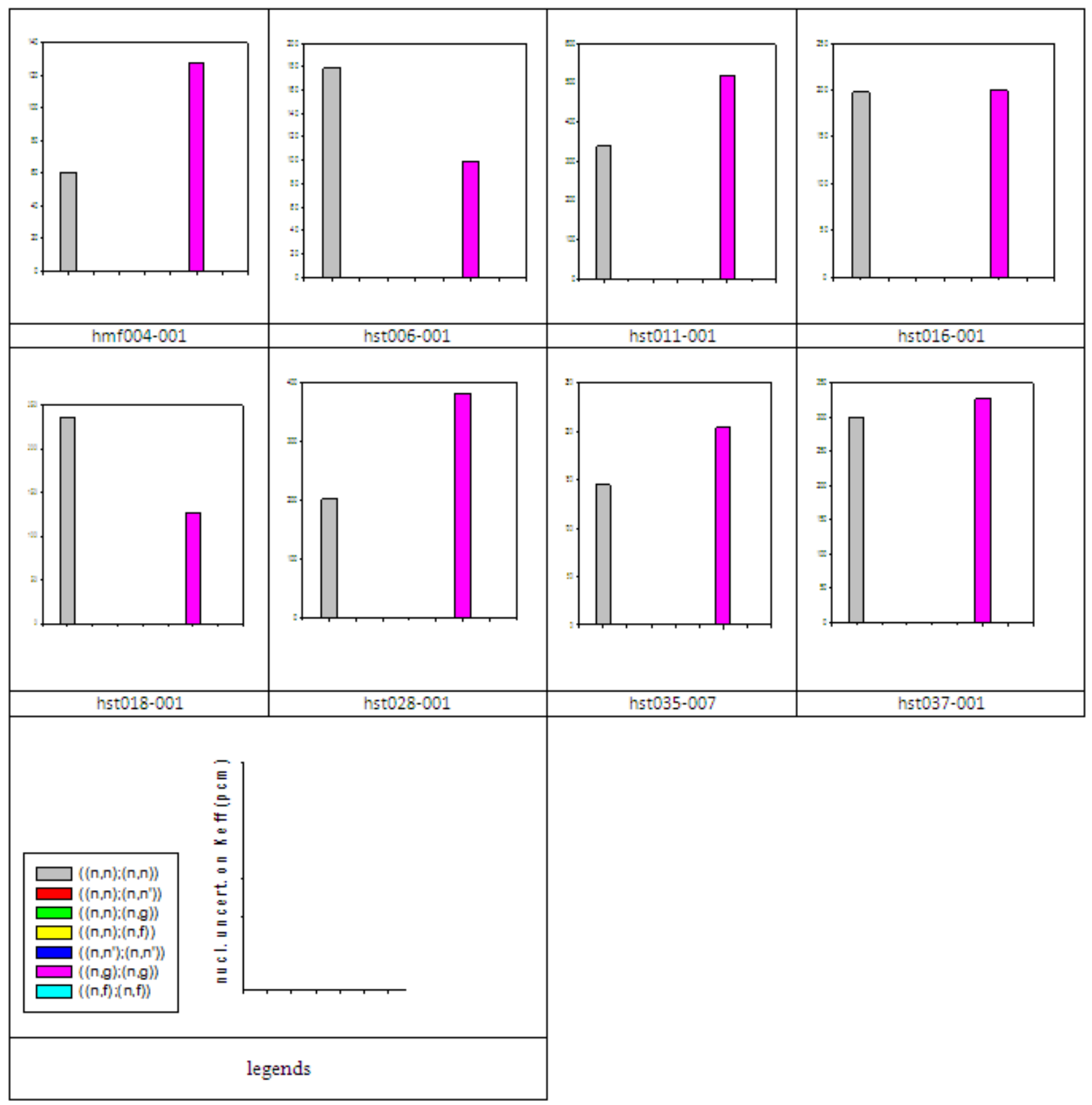

Figure 9. Uncertainties (pcm) produced by different cross sections of ${ }^{1} \mathrm{H}$ for different experiences

\subsection{Interpretation and Conclusion on the Results of ${ }^{1} \mathrm{H}$}

- The cross sections uncertainties have no effect on the effective multiplication factor (Keff) in all experiences, except the elastic scattering and the capture cross sections uncertainties have the great contribution on the Keff uncertainties in the thermal experiences and in one rapid experience. Then, the elastic scattering and the capture cross sections and their covariance matrices lack the adjustment especially in the thermal experiences.

\section{Conclusion}

In this work we have analysed the sensitivities and uncertainties on the effictive multiplication factor (Keff) produced by ENDF/B-VII.0 nuclear data; espicially the elastic and inelastic scattering, capture and fission cross sections and their correlations in the ${ }^{1} \mathrm{H},{ }^{16} \mathrm{O},{ }^{235} \mathrm{U}$ and ${ }^{238} \mathrm{U}$ by the adjoint- weigted technique of the MCNP6 code. Firstly, we validated our perturbation technique in four experiences. After, a series of critical experiences have been studied by using the Monte Carlo code MCNP6 and the ERRORJ module of the last update NJOY99 to calculate the sensitivities vectors and to process the covarionce matrices. In the end, the sensitivity vectors and covariance matrices are combined in order to obtain final uncertainties. As a conclusins:

- The four cross sections (elastic, inelastic, capture and 
fission) of the ${ }^{235} \mathrm{U}$ and their covariance matrices Lack the adjustment especially in the rapid energies.

- The elastic cross section of the ${ }^{16} \mathrm{O}$ and its covariance matrice lack the adjustment especially in the thermal energies.

- The elastic and capture cross sections of the ${ }^{1} \mathrm{H}$ and their covariance matrices lack the adjustment especially in the thermal energies.

The adjustment of these cross sections and their covariance matrices is our aim in our future works.

\section{REFERENCES}

[1] Chadwick, M.B., et al., 2006. ENDF/B-VII.0: Next Generation Evaluated Nuclear Data Library for Nuclear Science and Technology, Nuclear data Sheets,vol. 107, pp.2931-3060. http://www.nndc.bnl.gov/exfor7/endf00.htm. (july,2013).

[2] D. B. Pelowitz: MCNP User's Manuel, Los Alamos National Laboratory, December 2011, LA-CP-11-01708.

[3] G.W. McKinney, "Verification of the Monte Carlo differential operator technique for MCNP”, LA-13098.

[4] B. C. Kiedrowski and F. B. Brown, "Adjoint-Weighting for Critical Systems with Continuous Energy Monte Carlo," Proceedings of the 2009 Nuclear Criticality Safety Division (of the American Nuclear Society) Topical Meeting on Realism, Robustness, and the Nuclear Renaissance, Richland, Washington, September 13-17, CD-ROM (2009).

[5] B. C. Kiedrowski, F. B. Brown, and P. P. H. Wilson, "Adjoint-Weighted Tallies for k-Eigenvalue Calculations with Continuous-Energy Monte Carlo," Nuclear Science and Engineering, 168, 3, 226-241 (2011).

[6] B.C. Kiedrowski \& F.B. Brown, "Comparison Of The Monte Carlo Adjoint-Weighted And Differential Operator Perturbation Methods", SNA+MC-2010, Tokyo, Oct 17-20 [also, LA-UR-10-05215] (2010).

[7] C. LAVILLE, "Etude de différentes méthodes de calculs de coefficients de sensibilités du Keff aux données nucléaires", Université de Montréal, 2011.

[8] M.L. Williams, B.T. Rearden, "SCALE-6 Sensitivity/Uncertainty Methods and covariance Data",Science Direct, Nuclear Data sheets 109(2008) 2796-2800.

[9] T. IVANOVA, I. DUHAMEL and E. LETANG, Impact of cross section covariance Data on Results of High-confidence criticality validation, Journal of the Korean Physical Society, Vol. 59, $\mathrm{N}^{\circ}$ 59, August 2011, pp. $1170 \sim 1173$.

[10] International Evaluation Co-operation. Volume 29, "Uranuim-235 capture cross section in the $\mathrm{Kev}$ to $\mathrm{Mev}$ energy region", NEA/NSC/WPEC/DOC (2011)433.

[11] C.J. Diez, J.J. Herrero, O. Cabellos, and J.S. Martinez, "Propagation of Cross Section Uncertainties Criticality Calculations in the Framework of UAM- Phase I Using MCNP-2.7e and SCALE-6.1. Hindawi Publishing cooperation, Science and Technology of Nuclear installations, Volume 2013, Article ID 380284, 10 pages. http://dx.doi.org/10.1155/2013/380284.

[12] International Handbook of Evaluated Criticality Safty Benchmark Experiments, NEA/NSC/DOC (95)03/III, September 2007 Edition.

[13] R. E. MacFarlane,2002, NJOY99 Nuclear Data Processing system.[online]

Available:http://t2.lanl.gov/nis/codes/njoy99/index.html ,(jul $\mathrm{y}, 2013)$

[14] Shibata K. et. al. "JENDL-4.0: A New Library for Nuclear Science and Engineering", Nuclear Science and Technology, Vol. 48 (1), pp. 1-30, (2011).

[15] M. Kaddour et. Al. "Impact of the atomic density on the uncertainties of the effective multiplication factor due to nuclear data uncertainties ", International Journal of Innovation and Applied studies ISSN 2028-9324 Vol. 4 No. 1 sep. 2013, pp. 171-181. 